\title{
STUDY OF METALLO-B-LACTAMASE PRODUCTION IN CLINICAL ISOLATES OF PSEUDOMONAS AERUGINOSA IN A TEACHING HOSPITAL OF RURAL GUJARAT - INDIA
}

Yagnesh Pandya, H.H. Agravat.

1. Associate Professor, Department. of Microbiology, P.S. Medical College, Karamsad.

2. Dean, Department. of Microbiology, C.U, Shah Medical College, Surendranagar.

\section{CORRESPONDING AUTHOR:}

Dr. Yagnesh Pandya.

Associate Professor,

Dept. of Microbiology,

P.S. Medical College,

Karamsad, Dist Anand Gujarat, India.

E-mail: yagneshgp@charutarhealth.org

ABSTRACT: BACKGROUND: Pseudomonas aeruginosa exhibits intrinsic resistance to a variety of antimicrobials including beta lactams. Imipenem-resistant Pseudomonas aeruginosa resulting from metallo- $\beta$-lactamases production represent a significant and rapidly emerging problem and threat in most part of the world. Detection of metallo- $\beta$-lactamase (MBL)-producing Pseudomonas aeruginosa is crucial for the optimal antibiotic treatment of the seriously ill patients. AIM OF THE STUDY: The study was undertaken to detect Metallo- $\beta$-lactamases among clinical isolates of Pseudomonas aeruginosa in the multidisciplinary tertiary care 520-bedded hospital. MATERIAL AND METHODS: Two hundred eighty three consecutive clinical isolates of Pseudomonas aeruginosa were subjected to susceptibility testing by disc-diffusion assay and mini API system (bioMerieux-France). Imipenem resistant Pseudomonas aeruginosa isolates were studied for metallo- $\beta$-lactamase production by IPM-EDTA-disk synergy test. RESULTS: Imipenem- resistance by disc diffusion method was found in 35 out of 283 isolates (12.3\%) of Pseudomonas aeruginosa. Of the 35 imipenem-resistant isolates 30 (85.7\%) were Metallo-beta-lactamases producers with average zone diameter difference of $16.5 \mathrm{~mm}$ between imipenem disk and imipenem plus EDTA disk. Of 35 imipenem-resistant isolates, 21(60\%) were from ICU patients and 14(40\%) were from Wards. CONCLUSION: IPM-EDTA-disk synergy test can be used as a suitable screening method in the clinical microbiology laboratory. Presence of Metallo- $\beta$-lactamase production among imipenem-resistant Pseudomonas aeruginosa was high in our institution which is disturbing and forces us for prompt infection control measures which are well-organized and continued. Combined approaches of rotational antibiotic therapy and training programmes for the health care workers might be beneficial to fight against these multi drug resistant pathogens.

KEY WORDS: Metallo-beta-lactamase, Pseudomonas aeruginosa, Imipenem, Multi drug resistance

INTRODUCTION: Pseudomonas aeruginosa is a classic opportunist pathogen with intrinsic resistant to many antibiotics and disinfectants. [1] This organism survives in various environments in nature and in homes and hospitals. Because of the ubiquitous nature of Pseudomonas aeruginosa, transmission of this organism to humans can occur in variety of ways. [2] It is 


\section{ORIGINAL ARTICLE}

physiologically versatile and flourishes as a saprophyte in warm moist situations in the human environment, including sink, drains, respirators, humidifiers and disinfectant solutions. [1] Increasing incidence of Pseudomonas aeruginosa causing serious nosocomial infections in hospital intensive care units are being reported worldwide. It is commonly associated with infections such as bacteremia, urinary tract infection, meningitis, skin and soft tissue infections and pneumonia with high mortality rates in hospitalised patients. Among the $\beta$-lactams, carbapenems are effective agents for treatment of serious gram-negative bacterial infections. These antibiotics are well-suited to this use because of their broad spectrum activity and resistance to hydrolysis by most $\beta$-lactamases, including the extended-spectrum $\beta$-lactamases (ESBL). [3] Metallo- $\beta$-lactamase (MBL) production is increasingly reported as a cause of high-level Carbapenem resistance among Pseudomonas aeruginosa, an important nosocomial multi-drug resistance pathogen. $[4,5]$

Carbapenemases are $\beta$-lactamases, which includes serine- $\beta$-lactamases (KPC, OXA) and metallo- $\beta$ lactamases (MBLs). The latter require metal ion zinc for their activity, which is inhibited by metal chelators like EDTA and thiol-based compound but not by sulbactam, tazobactam and clavulanic acid. Metallo- $\beta$-lactamases production is typically associated with resistance to aminoglycosides and fluoroquinolones leads to compromise in therapeutic options. bla-IMP and bla-VIM are most common metallo-beta-lactamases genes. The genes responsible for metallo- $\beta$-lactamases production may be chromosomal or plasmids mediated and pose a risk of transfer among other Gram-negative bacteria. [6]

Several phenotypic methods are described for the detection of metallo- $\beta$-lactamases producing bacteria. All these methods are based on the ability of the metal chelators, such as EDTA and thiolbased compounds, to inhibit the activity of metallo- $\beta$-lactamases; but no standard guidelines are available.

The aim of the present study was to detect Metallo- $\beta$-lactamases among imipenem resistant clinical isolates of Pseudomonas aeruginosa.

MATERIALS AND METHODS: This study was prospective observational study. The study was conducted after the approval of the Human Research Ethics Committee (HREC) of the institution where the study was conducted. Various clinical specimens referred for bacteriological cultures from patients of all age groups and both sexes admitted in a tertiary care teaching hospital from January 2006 to January 2009 were included. The specimens comprised blood, urine, sputum, pus, endotracheal aspirate, CSF, swabs, etc. A total of 283 consecutive non-repetitive clinical isolates of Pseudomonas aeruginosa isolated and confirmed in Microbiology section of Central Diagnostic Laboratory. The isolates were identified by using mini API system, ID 32 GN (bioMerieux-France). The susceptibility of all isolates to 13 antimicrobial agents was determined using disc-diffusion assay on Muller-Hinton agar medium (Hi-media Laboratories, Mumbai) and ATB PSE 5 strip (bioMerieux-France) and the results were interpreted according to the CLSI guidelines. [7] Paper discs (Hi-media Laboratories, Mumbai) impregnated with ceftazidime (30 $\mu \mathrm{g})$, ciprofloxacin (5 $\mu \mathrm{g})$, imipenem $(10 \mu \mathrm{g})$, gentamicin $(10 \mu \mathrm{g})$, amikacin $(30 \mu \mathrm{g})$, cefepime $(30 \mu \mathrm{g})$, piperacillin $(100 \mu \mathrm{g})$, piperacillin-tazobactam $(100 / 10 \mu \mathrm{g})$, tobramycin $(10 \mu \mathrm{g})$, aztreonam $(30 \mu \mathrm{g})$, meropenem $(10 \mu \mathrm{g})$, ticarcillin $(75 \mu \mathrm{g})$, norfloxacin $(10 \mu \mathrm{g})$ (in urinary isolates) were used in drug susceptibility testing of isolates and multidrug resistant strains were identified [Figure: 1]. Pseudomonas aeruginosa ATCC 27853 used as quality control strain. 


\section{ORIGINAL ARTICLE}

DETECTION OF METALLO-B-LACTAMASE: IPM-EDTA-disk synergy test [8] : To make 0.5 M EDTA solution $186.1 \mathrm{~g}$ of disodium EDTA was dissolved in $1000 \mathrm{ml}$ of distilled water and $\mathrm{pH}$ was adjusted to 8.0 by using $\mathrm{NaOH}$. EDTA imipenem disks were prepared by adding EDTA solution to $10-\mu \mathrm{g}$ imipenem disks to obtain a concentration of $750 \mu \mathrm{g}$. The disks were dried immediately in an incubator and stored at $4^{\circ} \mathrm{C}$ or at $-20^{\circ} \mathrm{C}$ in an air tight vial. Test strains were adjusted to the McFarland 0.5 standard and checked with densitometer (bioMerieux-France) and were inoculated to Mueller Hinton agar. A 10- $\mu$ g-imipenem disk and an imipenem plus $750 \mu$ g EDTA were placed on Mueller Hinton agar. Another disk containing only $750 \mu \mathrm{g}$ EDTA was also placed as a control. After overnight incubation, the established zone diameter difference of $\geq 7 \mathrm{~mm}$ between imipenem disk and imipenem plus EDTA was interpreted as EDTA synergy positive. [9]

RESULTS: In the present study the total number of clinical specimens processed in the Microbiology laboratory was 8320 during the study period, out of which 3120 (37.5\%) were found to be culture positive. Out of 3120 culture positive isolates, $283(9.07 \%)$ were confirmed as Pseudomonas aeruginosa. Of these 283 isolates, 132(46.6\%) were obtained from pus and the remainder from endotracheal secretion 76 (26.8\%), sputum 33 (11.6\%), urine 25(8.8\%), blood 10 (3.5\%), broncho-alveolar lavage 4 (1.4\%) and pleural fluid 3 (1.06\%). Out of 283 Pseudomonas aeruginosa isolates 164 (57.9\%) were isolated from clinical specimens of different wards followed by different intensive care units 119 (42.04\%). The most common specimen from different wards, 96 (58.5\%) was pus, followed by sputum 24 (14.6\%) and urine 17 (10.36\%). Out of 96 pus specimens, 43 (47.9\%) were from orthopaedic ward, followed by $28(29.16 \%)$ were from general surgery ward. Out of 164 isolates, 46 (28.04\%) were from orthopaedic ward followed by 39 $(23.78 \%)$ from general surgery ward. The most common specimen from intensive care units, 66 (55.46\%) was endotracheal secretion, followed by pus 18 (15.12\%). Out of 66 endotracheal secretion specimens, 33 (50\%) were from surgical ICU, followed by 17 (27.75\%) from medical ICU. Out of 119 total isolates, 51 (42.85\%) from surgical ICU, followed by 30 (25.21\%) from medical ICU.

Resistance pattern of Pseudomonas aeruginosa indicating high prevalence of MDR as shown in Table: 1. Imipenem- resistance by disc diffusion method was found in 35/283 (12.3\%) isolates of Pseudomonas aeruginosa and all were MDR. Of 35 imipenem-resistant isolates, $21(60 \%)$ isolates from ICU patients and $14(40 \%)$ isolates were from different wards. All 35 imipenem-resistant isolates were subjected to IPM-EDTA- synergy test. The test was found positive for metallo- $\beta$ lactamases in 30 out of 35 isolates $(85.7 \%)$ with average zone diameter difference between imipenem disk and imipenem plus EDTA disk for metallo- $\beta$-lactamase-positive isolates of $16.5 \mathrm{~mm}$. 


\section{ORIGINAL ARTICLE}

\begin{tabular}{|lcc|}
\hline \multicolumn{2}{|l|}{ Table 1: Antibiotics susceptibility pattern of all isolates and MBL producer } \\
\hline Antimicrobial agents & $\begin{array}{c}\text { \% of resistance } \\
\text { of all isolates } \\
(\mathrm{n}=253)\end{array}$ & $\begin{array}{c}\text { \% of resistance of } \\
\text { MBL producing strains } \\
\text { (n=30) }\end{array}$ \\
\hline Ceftazidime & 60.0 & 89.4 \\
\hline Gentamicin & 67.2 & 100 \\
\hline Tobramycin & 61.6 & 94.7 \\
\hline Piperacillin & 51.9 & 81.5 \\
\hline Amikacin & 58.2 & 97.3 \\
\hline Aztreonam & 70.2 & 100 \\
\hline Cefepime & 68.9 & 97.3 \\
\hline Ciprofloxacin & 62.5 & 100 \\
\hline Imipenem & 12.3 & 100 \\
\hline
\end{tabular}

DISCUSSION: Nosocomial outbreaks of carbapenem-resistant Pseudomonas aeruginosa and Acinetobacter spp. due to metallo- $\beta$-lactamases (MBLs) production have been reported from different parts of the world. [10,11] The emergence of these metallo- $\beta$-lactamases in gram negative bacilli is becoming a therapeutic challenge as these enzymes possess high hydrolytic activity that leads to degradation of cephalosporins; the treatment options are not available, or costly/unsafe with pitiable results. ${ }^{[12]}$

We found that the most common nosocomial pathogen associated with infection in intensive care units was Pseudomonas aeruginosa. Among all bacterial isolates, Pseudomonas aeruginosa was found to be the most common in respiratory tract infections and surgical site infections. The ability of patients in intensive care units to ward off infections is seriously compromised. Natural host defence mechanisms may be impaired by underlying diseases or as a result of medical or surgical interventions. All patients admitted to intensive care units will have at least one, often several, indwelling devices that break the normal skin barriers and establish direct access between the external environment and normally sterile body sites.

By using the Imipenem-EDTA disk method, a very high percentage of imipenem resistant Pseudomonas aeruginosa (85.7\%) isolates showed metallo- $\beta$-lactamase production. This finding is consistent with other reports, ${ }^{[13]}$ giving the support that acquired metallo- $\beta$-lactamases can speedily emerge and set up a condition of endemicity in certain situations . [9] A majority of our isolates also showed resistance to other important antipseudomonal groups of antibiotics [Table:1], which is a feature of majority of metallo- $\beta$-lactamases producing isolates.[14] $A$ number of metallo- $\beta$-lactamase producers in Pseudomonas aeruginosa showed significant difference between zone of inhibition by imipenem and imipenem plus EDTA disk. When compared with non- metallo- $\beta$-lactamase producers the average zone difference for the metallo- $\beta$-lactamase producers was $16.5 \mathrm{~mm}$ versus $4 \mathrm{~mm}$ for the non- metallo- $\beta$-lactamase producers, making this test dependable for the initial screening of metallo- 


\section{ORIGINAL ARTICLE}

$\beta$-lactamase production in clinical isolates in routine diagnostic laboratories. This finding is consistent with other report. [9]

Reports from various parts of the world showing emergence of metallo- $\beta$-lactamase enzymes in Enterobacteriaceae ${ }^{[15]}$ is an evidence for the spread of these enzymes in this family. New Delhi metallo- $\beta$-lactamases (NDM) is a nomenclature that is designated for carbapenemases found in Enterobacteriaceae isolated from patients in the United Kingdom and elsewhere who have had healthcare contact in India or Pakistan. [16]

Metallo- $\beta$-lactamase producing Pseudomonas aeruginosa in our clinical isolate is frightening and reflects unwarranted use of carbapenem. Timely detection and prompt instillation of infection control measures are vital to prevent further spread of metallo- $\beta$-lactamase to other Enterobacteriaceae. It is also important to have and follow the stringent antibiotic policy of the hospital to avoid unnecessary use of antibiotics. Over all incidence of Pseudomonas aeruginosa as a nosocomial pathogen in our hospital is low but they are predominantly multidrug resistance. Antimicrobial resistance is varying considerably among different countries, different hospitals, and among different areas of the same hospitals; therefore surveillance studies in the different areas of the hospital are needed to decide most suitable therapy. Occurrence of metallo- $\beta$-lactamase producing Pseudomonas aeruginosa isolates poses not only a treatment problem but also a serious concern for infection control measures. There are multiple mechanisms that cause multidrug resistance in Pseudomonas aeruginosa; for the development of new treatment protocols, it is crucial to know the interaction of various resistance mechanisms.

To conclude, the percentage of multidrug resistance Pseudomonas aeruginosa was high in our institution which is disturbing and forces us for prompt infection control measures which are wellorganized and continued. An active infection control programme, with periodic surveillance of infection and hospital environment, can effectively implement the guidelines for prevention of nosocomial infections to improve patient safety.

\section{REFERENCES:}

1. Collee JG, Diguid JP, Fraser AG. Pseudomonas, Stenotrophomonas, Burkholderia. In:Mackie and McCartney practical Medical Microbiology. 14 th ed. (Churchill Livingstone, Edinburgh) 1996:41324

2. Betty A. Forbes, Daniel F. S., Alice S.W. Bailey and Scott's Diagnostic Microbiology, $12^{\text {th }}$ Edi. Mosbey Elsevier 2007.

3. Bush K, Jacoby GA, Medeiros AA. A functional classification scheme for $\beta$-lactamases and its correlation with molecular structure. Antimicrobial Agents Chemother. 1995; 39:1211-33.

4. Livermore DM. Multiple mechanisms of antimicrobial resistance in Pseudomonas aeruginosa: our worst nightmare? Clin Infect Dis. 2002; 34:634-40.

5. Sader HS, Reis AO, Silbert S, Gales AC. IMPs, VIMs and SPMs: the diversity of metallo- $\beta$-lactamases produced by carbapenem-resistant P.aeruginosa in a Brazilian hospital. Clin Microbiol Infect. 2005; 11:73-76.

6. Purohit M, Mendiratta D K, Deotale V S, Madhan M, Manoharan A, Narang P. Detection of metallo- $\beta$-lactamases producing Acinetobacter baumannii using microbiological assay, disc synergy test and PCR. Indian J Med Microbiol [serial online] 2012 [cited 2013 Apr 10]; 30:45661. 


\section{ORIGINAL ARTICLE}

7. Clinical and Laboratory standards institute (CLSI), performance standards for antimicrobial susceptibility testing, $18^{\text {th }}$ informational supplements CLSI Document M 100-518 January 2008.

8. Yong D, Lee K, Yum JH, Shin HB, Rossolini GM, Chong Y. Imipenem-EDTA disk method for differentiation of metallo-beta-lactamase-producing clinical isolates of Pseudomonas spp. and Acinetobacter spp. J Clin Microbiol 2002;40:3798-801

9. Irfan S, Zafar A, Guhar D, Ahsan T, Hasan R. Metallo- $\beta$-lactamase-producing clinical isolates of Acinetobacter species and Pseudomonas aeruginosa from intensive care unit patients of a tertiary care hospital. Indian J Med Microbiol 2008;26:243-45

10. Ohara M, Kouda S, Onodera M, Fujiue Y, Sasaki M, Kohara T, et al. Molecular characterization of imipenem resistant Pseudomonas Aeruginosa in Hiroshima, Japan. Microbiol Immunol 2007;51:271-77

11. Peleg AY, Franlin C, Bell JM, Spelman DW. Dissemination of metallo-beta-lactamase gene bla IMP-4 among gram negative pathogens in a clinical setting in Australia. Clin Infect Dis 2005;41:154956

12. Marra AR, Pereira CA, Gales AC, Menezes LC, Cal RG, de Souza JM, et al. Blood stream infections with metallo-beta-lactamase producing Pseudomonas aeruginosa : Epidemiology, microbiology and clinical outcomes. Antimicrob Agents Chemother 2006; 50:388-90.

13. Lagatolla C, Tonin EA, Monti-Bragadin C, Dolzani L, Gombac F, Bearzi C, et al . Endemic Carbapenem-resistant Pseudomonas aeruginosa with acquired metallo-beta-lactamase determinants in a European hospital. Emerg Infect Dis 2004; 10:535-38.

14. Pitout JD, Gregson DB, Poirel L, McClure JA, Le P, Church DL. Detection of Pseudomonas aeruginosa producing metallo-beta-lactamases in a large centralized laboratory. J Clin Microbiol 2005; 43:3129-35.

15. Ikonomidis A, Tokatlidou D, Kristo I, Sofianou D, Tsakris A, Mantzana P, et al. Outbreaks in distinct regions due to a single Klebsiella pneumoniae clone carrying a bla VIM-1 metallo- betalactamase gene. J Clin Microbiol 2005; 43:5344-47.

16. Krishna B. New Delhi metallo-beta-lactamases: A wake-up call for microbiologists. Indian J Med Microbiol 2010;28:265-66 


\section{ORIGINAL ARTICLE}

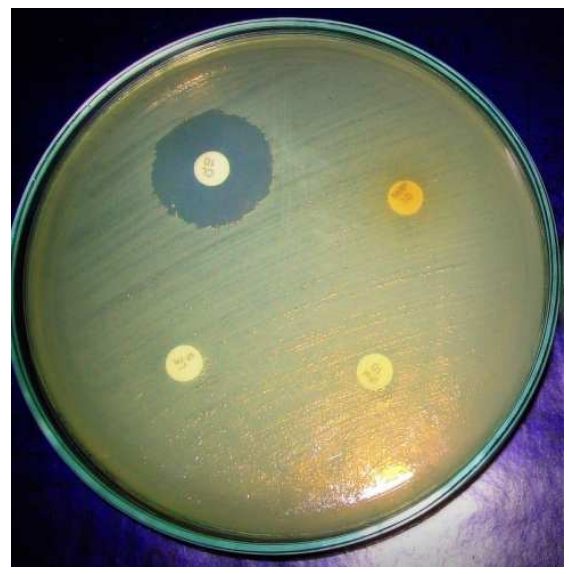

Figure 1: Multidrug resistant strain of Pseudomonas aeruginosa

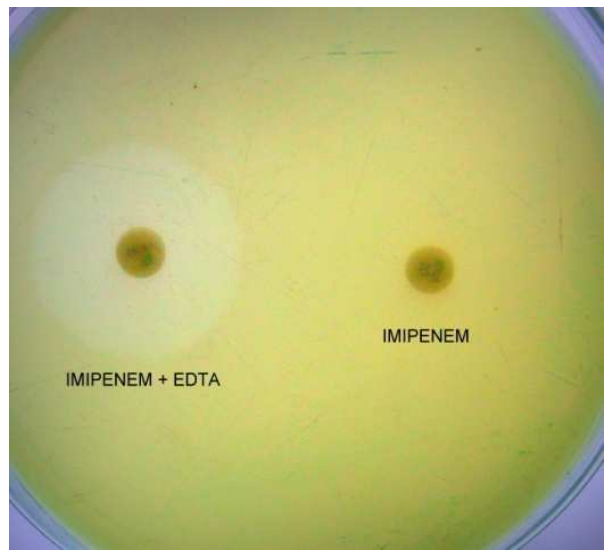

Figure: 2 IPM-EDTA-disk synergy test 